\title{
Understanding Health Information Technology Induced Medication Safety Events by Two Conceptual Frameworks
}

\author{
Ju Wang ${ }^{1}$ Hongyuan Liang ${ }^{2}$ Hong Kang ${ }^{1}$ Yang Gong ${ }^{1}$ \\ ${ }^{1}$ School of Biomedical Informatics, University of Texas Health Science \\ Center, Houston, Texas, United States \\ 2 Department of Radiology, Shengjing Hospital of China Medical \\ University, Shenyang, Liaoning, China \\ Address for correspondence Yang Gong, MD, PhD, School of \\ Biomedical Informatics, University of Texas Health Science Center, \\ 7000 Fannin Street, Suite 600, Houston, TX 77030, United States \\ (e-mail: yang.gong@uth.tmc.edu; gongyang@gmail.com).
}

Appl Clin Inform 2019;10:158-167.

\section{Abstract}

Keywords

- patient safety

- health information systems

- medication errors
Background While health information technology (health IT) is able to prevent medication errors in many ways, it may also potentially introduce new paths to errors. To understand the impact of health IT induced medication errors, this study aims to conduct a retrospective analysis of medication safety reports.

Methods From the U.S. Food and Drug Administration (FDA) Manufacturer and User Facility Device Experience database, we identified reports in which health IT is a contributing factor to medication errors. We applied two conceptual frameworks, Sittig and Singh's sociotechnical model and Coiera's information value chain, to examine the identified reports.

Results We identified 152 unique reports on health IT induced medication errors as the final report set for review. The majority (65.13\%) of the reports involved multiple contributing factors according to the sociotechnical model. Three dimensions, that is, clinical content, human-computer interface, and people, were involved in more reports than the others. The transition of the effects of health IT on medication practice was summarized using information value chain. Health IT related contributing factors may lead to receiving wrong information, missing information, receiving partial information and delayed information, and receiving wrong information and missing information tend to cause the commission errors in decision-making.

Conclusion The two frameworks provide an opportunity to understand a comprehensive context of safety event and the impact of health IT induced errors on medication safety. The sociotechnical model helps identify the aspects causing medication safety issues. The information value chain helps uncover the effect of the health IT induced medication errors on health care process and patient outcomes.

\section{Background and Significance}

Medication errors, including unsafe medication practices, are a leading cause of avoidable harm in health care systems across the world. ${ }^{1}$ By estimate, millions of preventable adverse medication events occur each year in the United States. ${ }^{2}$ Medication practices encompass the continuum of prescribing followed by transcribing, preparing and dispen- sing, administering, and monitoring and involve multiple players such as clinicians, pharmacists, nurses, and patients. ${ }^{2}$ The risk of error exists in each of these steps, where health care professionals make decisions.

Health information technology (health IT) is broadly defined as the use of information and communication technology to collect, transmit, display, or store clinical data in health care to support the delivery of patient or population received

September 21, 2018

accepted after revision

January 7, 2019 (c) 2019 Georg Thieme Verlag KG Stuttgart · New York
DOI https://doi.org/

10.1055/s-0039-1678693. ISSN 1869-0327. 
care or to support patient self-management. ${ }^{3}$ The adoption of health IT has brought in many benefits to enhance medication safety. Its main classes of strategies for preventing errors and adverse events include improving communication, making knowledge more readily accessible, retrieving key pieces of information, assisting calculations, real-time checking, assisting monitoring, and providing decision support. ${ }^{4}$ For example, processing a medication prescription order through a computerized provider order entry (CPOE) system decreases the likelihood of error on that order. $^{5}$ Use of the barcode electronic medication administration system (e-MAR) substantially reduces the rate of errors in the aspects of order transcription and medication administration, as well as potential adverse drug events. ${ }^{6,7}$ Handheld devices can assist clinicians expeditiously in retrieving drug knowledge at the point of care and making drug-related decisions. ${ }^{8} \mathrm{~A}$ computer-assisted dose calculator can streamline medication ordering and administration toward safer health care. ${ }^{9}$

While health IT is widely regarded as the technical solution for enhancing medication safety, it may also potentially introduce new paths to errors. ${ }^{10}$ Since health IT products have become pervasive in health care delivery, the potential risks related to health IT have increased significantly. Health IT has been considered a contributing factor to patient safety events ${ }^{11}$ including medication errors. The lack of interoperability among hospital information systems limits the performance of health IT products, which may cause delayed or inadequate information access for making right decisions at the right time and lead to adverse medication events. ${ }^{12,13}$ Unexpected software design issues and inappropriate use of CPOE products may delay medication orders and thus cause unexpected disruptions in health care delivery. ${ }^{14}$ Poorly designed CPOE user interface (e.g., drug name display) may result in false medication selection during prescription. ${ }^{15}$ Malfunction of data transmission in e-prescribing systems has the risk of delivering wrong medication to a patient. ${ }^{16}$ While medication-related alerts in clinical decision support systems often lead to alert fatigues, inappropriate alert overrides may post preventable harm to patients. ${ }^{17,18}$

An effective way to prevent errors is to learn from patient safety events, including unsafe conditions, near misses and incidents. Many event-reporting systems have been created and put in use for learning purpose, which enables patient safety specialists to analyze events, identify underlying factors, and generate actionable knowledge to mitigate risks. ${ }^{19}$ By mining exclusive or publicly accessible event databases, researchers have attempted to quantify and classify the impact of health IT on health care in general or on medication safety in specific. Magrabi et al examined patient safety events that were reported by public hospital clinicians across a state of Australia and developed a classification of computer use problems where human factors were distinguished from machine-related problem. ${ }^{20}$ The classification was further expanded by analyzing health IT induced events from the U.S. Food and Drug Administration (FDA) Manufacturer and User Facility Device Experience (MAUDE) data- base. ${ }^{21}$ With the adoption of Magrabi's classification, a study analyzed a large sample of health IT related medication errors reported by health care professionals in community pharmacies and hospitals in the Netherlands. ${ }^{22}$

The report of the Institute of Medicine Committee, titled Health IT and Patient Safety: Building Safer Systems for Better Care, recognized that health IT is part of a complex sociotechnical system..$^{23}$ Health IT products play roles in the context that is "sociotechnical" in nature, involving interaction between technology, people, processes, organizations, and the external environment. ${ }^{11}$ Toward addressing the sociotechnical challenges of IT products within complex adaptive health care systems, Sittig and Singh proposed an eight-dimensional sociotechnical model. ${ }^{24}$ The sociotechnical model has been adopted in analyzing electronic health record (EHR) related investigation reports from health care facilities within the Department of Veterans Affairs. ${ }^{25}$ Guided by the sociotechnical model, a composite classification of health IT related contributing factors was created and further used to investigate the health IT related sentinel events reported to the Joint Commission. ${ }^{11}$

To prevent medication errors, health care professionals need to understand event occurrence, root causes, contributing factors, and potential intervention in the medication practice workflow. Sittig and Singh's sociotechnical model offers a broad view of health IT related errors from the sociotechnical perspective. Unfortunately, the view does not extend to impact of the errors, for example, altered care processes, extended stays, or delayed discharges in hospital. The information value chain conceptual model proposed by Coiera connects the use of health IT to the final health care outcome, ${ }^{26}$ for example, the consequences of medication errors in the scenario of medication practice. A recent systematic review based on the information value chain model studied reporting problems of health IT and their effects on care delivery and patient outcomes. ${ }^{27}$ It is imperative to quantify risks and benefits with regard to patient safety and systematically identify issues from large and publically accessible resources. The information value chain, when used in conjunction with the sociotechnical model for health IT related errors, can enhance measurement and facilitate identification of the most significant risks to patient safety. This is our initial step in applying two conceptual frameworks to describe how health IT fails to work as intended or designed, especially how it fails to prevent medication safety events, where interactions between health IT and clinicians are intensive. Despite both frameworks have been successfully applied in analyzing health IT related patient safety events in separate investigations, the events with a combined view could bring us a new perspective to health IT induced medication errors.

\section{Objective}

This study aims to conduct a retrospective analysis of safety reports and reveal a new perspective to health IT induced medication errors through the application of two conceptual frameworks. 


\section{Methods}

\section{Dataset}

In this study, we extracted safety reports on health IT induced medication errors from the FDA MAUDE database. Each year, the FDA receives several hundred thousand medical device reports (MDRs) of suspected device-associated deaths, serious injuries, and malfunctions. ${ }^{28}$ The MAUDE database houses these MDRs submitted by mandatory reporters from manufacturers and voluntary reporters from health care providers and patients. ${ }^{28}$ Though the MAUDE database is not dedicated to reporting health IT related safety events, it has received event reports involving health IT products. The MAUDE database is proven to be a useful source of information about the nature of software problems and their safety implications. ${ }^{21}$ The MAUDE database thus far contains up to 50,000 health IT related event reports, representing the most abundant and the only publicly accessible resource to extract such event reports. ${ }^{29}$

We retrieved 45,624 MAUDE reports that were submitted from 2008 to 2016 using a keyword-based filter. To reduce the review burden, $10 \%$ of the filtered reports were randomly sampled according to the distribution of Health Care Facility Association number, distributor number, and manufacturer number. A total of 3,521 reports were identified as health IT related reports. ${ }^{29}$ In this study, we further identified the ones relating to health IT induced medication errors, near misses, and unsafe conditions that may cause medication errors, and named them as health IT medication error (HIT-ME) reports. An HIT-ME report must meet the following criteria:

- The report is about a medication error or an unsafe condition that may cause a medication error.

- The term medication error refers to "any preventable event that may cause or lead to inappropriate medication use or patient harm while the medication is in the control of the health care professional, patient, or consumer."30

- Health IT is at least a contributing factor to the event.

- Any software malfunctions in EHR or a component of EHR (e.g., CPOE system, pharmacy system, e-MAR, etc.) that causes failure in retrieving, transmitting, processing/calculating, or storing information are considered health IT contributing factors.

- Any malfunctions of hardware necessary for running a health IT system are considered health IT contributing factors.

- Any health IT issues due to human factors such as humanmachine interactions (e.g., user's wrong operation of a software) are considered health IT contributing factors.

\section{Classification of Health IT Medication Error Reports}

Each report in the MAUDE database consists of structured fields and unstructured fields. The structured fields include "Brand Name," “MDR Report Key," "Type of Device," "Report Date," "Report Source," "Product Code," and so on; the unstructured fields usually consist of "Event Description" and "Manufacturer Narrative" if reported by a manufacturer. The "Event Description" describes the occurrence of the event, whereas the "Manufacturer Narrative" describes the response of the manufacturer to the event, the corresponding investigation, and the findings of the investigation if applicable.

All the reports are classified based on their structured fields of "Report Source" and "Product Code." The "Report Source" reflects how report quality varies across different reporters. The generic category of a device is identified by the "Product Code," which is assigned to a device based on the medical device product classification. The "Product Code" reflects which categories of health IT products tend to cause medication use issues. In this study, we analyzed the distribution of HIT-ME reports based on the classification.

\section{Conceptual Frameworks}

In this study, we examined all HIT-ME reports using two conceptual frameworks, that is, Sittig and Singh's sociotechnical model and Coiera's information value chain.

\section{Sittig and Singh's Sociotechnical Model}

To address the sociotechnical challenges involved in design, development, implementation, use, and evaluation of health IT within complex adaptive health care systems, Sittig and Singh proposed an eight-dimensional sociotechnical model $^{24}$ (- Table 1). It is emphasized that the eight dimensions are interdependent and interrelated concepts. The sociotechnical model laid a groundwork for defining, measuring, and analyzing health IT related safety hazards.

Under the eight dimensions of sociotechnical model, a composite classification of 77 health IT contributing factors was created using existing classifications, including the Agency for Healthcare Research and Quality (AHRQ) Common Formats, AHRQ Hazard Manager Ontology, and Magrabi's classification. ${ }^{11}$

\section{Coiera's Information Value Chain}

Based on the understanding that information has value, Coiera believes that an information value chain connects the act of seeking information in health care to the final patient outcome. ${ }^{26}$ The information value chain conceptual model aims to support the understanding of the effect of health IT on the process of health care delivery and the outcome. The information value chain connects the use of technology to the final outcome. The five steps of information value chain include interaction, information received, decision changed, care process altered, and outcome changed. At each step of the chain, there is potential for a value loss because not every input (new information) generates an output. The chain is initiated with an interaction with a health IT system and goes through several steps or subsequent interactions before outcomes are received. Subsequent interactions including information received, decision changed, care process altered, and outcome changed yield new information. However, only some of them may impact the next steps and eventually impact patient outcomes. ${ }^{26}$ If adding information to a process leads to improvement, the information must bring added value. Accordingly, if a health IT product does not function well or is used improperly, the information will bring value loss. For example, an e-MAR system induced problem might lead a pharmacist to receive wrong information about a prescription and, in turn, make a wrong decision 
Table 1 The 8 -dimension sociotechnical model and its operable explanation in reviewing health IT induced medication safety events

\begin{tabular}{|l|l|}
\hline Sociotechnical dimensions & Explanation \\
\hline $\begin{array}{l}\text { Hardware and software } \\
\text { computing infrastructure }\end{array}$ & $\begin{array}{l}\text { Equipment and software required to power, support, and operate clinical } \\
\text { applications and devices }\end{array}$ \\
\hline Clinical content & $\begin{array}{l}\text { Textual or numeric data, images, information, and knowledge entered, displayed, } \\
\text { processed, or transmitted }\end{array}$ \\
\hline Human-computer Interface & All aspects of the IT systems that users can see, touch, or hear as they interact with it \\
\hline People & $\begin{array}{l}\text { The humans involved in the design, development, implementation, and use of health IT, } \\
\text { including developers, health care providers, and patients }\end{array}$ \\
\hline Workflow and communication & $\begin{array}{l}\text { The processes and steps needed to ensure that patient care tasks are performed } \\
\text { when needed }\end{array}$ \\
\hline $\begin{array}{l}\text { Internal organizational } \\
\text { policies and procedures }\end{array}$ & $\begin{array}{l}\text { Internal culture, structures, policies, and procedures that affect all aspects of health } \\
\text { IT management and health care }\end{array}$ \\
\hline $\begin{array}{l}\text { External rules, regulations, } \\
\text { and pressures }\end{array}$ & $\begin{array}{l}\text { External forces that facilitate or place constraints on the design, development, } \\
\text { implementation, use, and evaluation of health IT in the clinical setting }\end{array}$ \\
\hline $\begin{array}{l}\text { System measurement } \\
\text { and monitoring }\end{array}$ & System availability, use, effectiveness, and unintended consequences of system use \\
\hline
\end{tabular}

Abbreviation: IT, information technology.

1. Identify event type according to $A H R Q$ classification

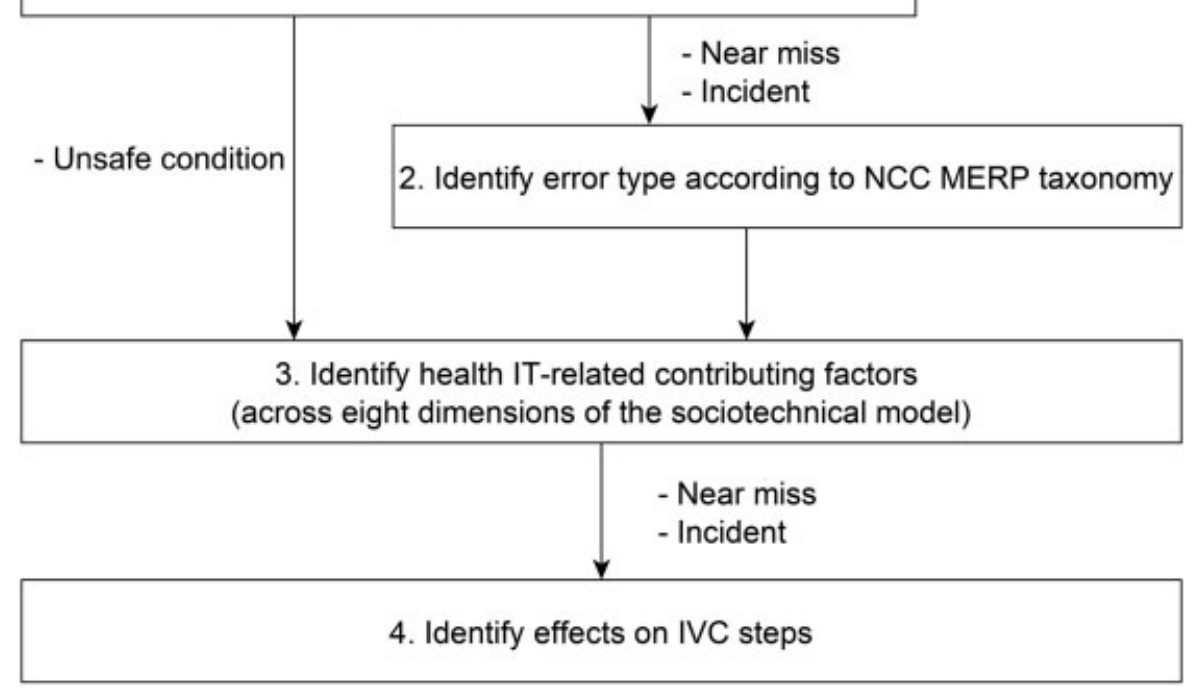

Fig. 1 The process of coding a health IT medication error report. AHRQ, Agency for Healthcare Research and Quality; IT, information technology; IVC, Coiera's information value chain; NCC MERP, National Coordinating Council for Medication Error Reporting and Prevention.

(e.g., dispensing wrong medication), and the medication may be incorrectly administered to a patient and lead to patient harm in the end. Kim et $\mathrm{al}^{27}$ improved the information value chain by assigning the types of health IT errors to the different stages of the value chain. For example, four types of errors in information were considered in the stage of information received: wrong, missing, partial, and delayed. We applied the improved value chain to display the distribution of medication errors on different error types.

\section{Expert Review}

In this study, each identified HIT-ME report from the MAUDE database was coded by an informaticist and a physician. All HIT-ME reports were coded according to the workflow shown in -Fig. 1. We went through the following steps:

- Identifying event type of a report according to the AHRQ Common Formats, a classification of reporting patient safety events, including incident, near miss, and unsafe condition. $^{31}$

- If event type is a near miss or an incident in medication practices, its error type will be revealed according to the National Coordinating Council for Medication Error Reporting and Prevention (NCC MERP) taxonomy, including dose omission, improper dose, wrong strength/concentration, wrong drug, wrong dosage form, wrong technique, wrong route of administration, wrong rate, 
wrong duration, wrong time, wrong patient, monitoring error, and deteriorated drug error. ${ }^{32}$

- Applying the sociotechnical model to identify health IT related contributing factors.

- Applying the information value chain model to identify the effect on the steps in the information value chain.

If the report was on an unsafe condition, we skipped steps 2 and 4 and applied only steps 1 and 3 . In some cases, an unsafe condition and an incident/near miss were both described in a single report. As a result, some reports were possibly annotated with multiple event types. Accordingly, multiple errors may occur in a single case; some reports were possibly annotated with multiple error types. A report was possibly annotated with multiple contributing factors relating to health IT as well. Besides, if a consensus cannot be reached as for any annotation between the two experts, a consultation was conducted with the third expert with health IT safety expertise.

\section{Results}

A Profile of Health IT Induced Medication Safety Events By reviewing the 9-year (2008-2016) sampled reports retrieved using a keyword-based filter, we manually identified 153 reports meeting the inclusion criteria of HIT-ME reports. One duplicate report was identified and excluded. Hence, the final HIT-ME report set for review contains 152 unique reports, among which 52 (34.21\%) were identified as unsafe conditions, 23 (15.13\%) as near misses, and 77 (50.66\%) as incidents.

\section{Analysis of Report Source}

In regard to report source, 29 (19.08\%) reports were "Manufacturer reports," indicating that the reports were submitted by manufacturers; 123 (80.92\%) reports were "Voluntary reports" and "User Facility reports," indicating that they were submitted by end users of corresponding health IT products, such as pharmacists, physicians, and patients. The average length of narratives in all HIT-ME reports was 187.26 words. A box plot in -Fig. 2 shows the length distribution. The narratives of the reports from man-

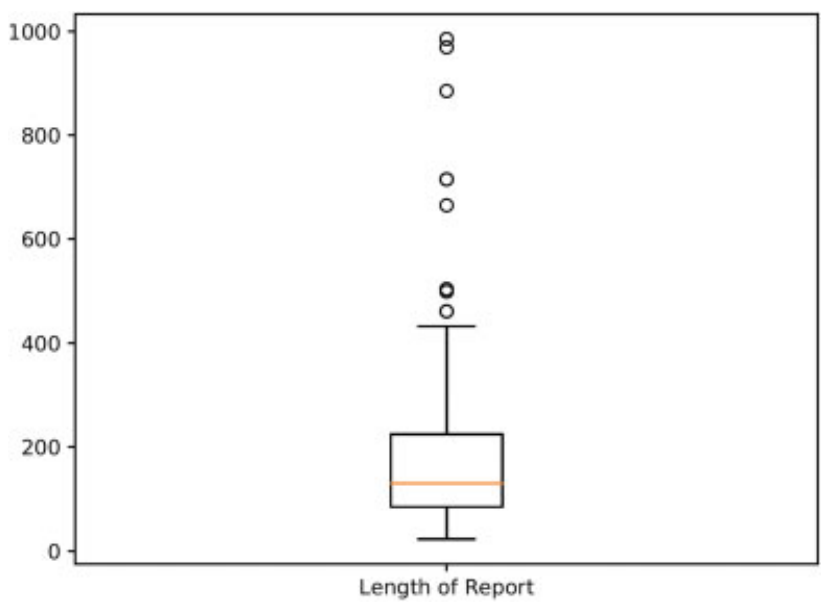

Fig. 2 Boxplot of the length of health information technology medication error reports. ufacturers usually consist of "event description" and "manufacturer narrative"; in contrast, the narratives of the reports from end users only contain "event description."

\section{Analysis of Product Code}

The 152 HIT-ME reports involved 23 types of medical devices ( - Table 2), the majority of which were related to information processing such as "software, transmission and storage, and patient data" (58; 38.16\%), "medical computers and software" (39; 25.66\%), and "calculator/data processing module, for clinical use" (14; 9.21\%). Nevertheless, a few types of medical devices that were not intuitively about information processing were also occasionally associated with the HIT-ME reports, such as "system/device, pharmacy compounding" and "pump, infusion, elastomeric."

\section{Analysis of Medication Error Types}

Among the 100 reports of near misses (23) and incidents (77), the error types of five reports failed to be identified due to the lack of information. The rest 95 reports cover 10 out of 13 (76.92\%) error types according to the NCC MERP taxonomy. The distribution of the involved error types is shown in - Fig. 3. "Improper dose," "wrong drug," and "wrong time" were the prevailing errors, whereas events with "wrong patient," "wrong dosage form," and "monitoring error" were uncommon. No event was categorized with "wrong technique," "wrong route of administration," and "deteriorated drug error" in the final HIT-ME report set.

\section{View of the Sociotechnical Model}

The contributing factors of three HIT-ME reports failed to be identified due to lack of information. We annotated 152 reports with the composite classification of health IT contributing factors under the sociotechnical model. Ninetynine $(65.13 \%)$ reports involved multiple contributing factors. - Fig. 4 shows that 152 reports cover 50 (64.93\%) out of 77 contributing factors in seven out of eight sociotechnical dimensions. Three dimensions, that is, clinical content, human-computer interface, and people, cover more reports than the others. Contributing factors associated with the dimensions of internal organizational policies and procedures, and workflow and communication were rarely identified. No contributing factor associated with system measurement and monitoring was found in this study.

\section{View through Information Value Chain}

Within the reports on near misses and incidents $(N=100)$, the effects of seven reports failed to be identified by the information value chain due to the lack of information. We visualized the transition of effects along with the information value chain manifested by the remaining 93 reports ( $\sim$ Fig. 5). The transition probability at each directed line denotes the probability of the transition from one effect to another. For example, the probability of "error-omission" in "decision changed" causing "error" in "care process" is 0.38 .

The transition of the effects of health IT on medication safety events was summarized from the viewpoint of information value chain (-Fig. 5). Health IT related contributing 
Table 2 FDA-coded medical devices involved in HIT-ME reports

\begin{tabular}{|c|c|c|}
\hline Product code & Medical device classification & No. of reports \\
\hline NSX & Software, transmission and storage, patient data & 58 \\
\hline LNX & Medical computers and software & 39 \\
\hline JQP & Calculator/data processing module, for clinical use & 14 \\
\hline OUG & Medical device data system & 10 \\
\hline $\mathrm{MMH}$ & Blood establishment computer software and accessories & 6 \\
\hline $\mathrm{NZH}$ & Medication management system, remote & 3 \\
\hline LHI & Set, IV fluid transfer & 2 \\
\hline $\mathrm{MUJ}$ & System, planning, radiation therapy treatment & 2 \\
\hline LLZ & System, image processing, radiological & 2 \\
\hline $\mathrm{MHX}$ & Monitor, physiological, patient (with arrhythmia detection or alarms) & 2 \\
\hline NXB & Dispenser, solid medication & 1 \\
\hline MEB & Pump, infusion, elastomeric & 1 \\
\hline NEP & System/device, pharmacy compounding & 1 \\
\hline BSZ & Gas machine, anesthesia & 1 \\
\hline NDC & Calculator, drug dose & 1 \\
\hline $\mathrm{DXJ}$ & Display, cathode-ray tube, medical & 1 \\
\hline MWI & Monitor, physiological, patient (without arrhythmia detection or alarms) & 1 \\
\hline IYE & Accelerator, linear, medical & 1 \\
\hline BRY & Cabinet, table and tray, anesthesia & 1 \\
\hline LZG & Pump, infusion, insulin & 1 \\
\hline NAY & System, surgical, computer-controlled instrument & 1 \\
\hline NVV & $\begin{array}{l}\text { Digital image, storage and communications, } \\
\text { nondiagnostic, Laboratory information system }\end{array}$ & 1 \\
\hline MSX & System, network and communication, physiological monitors & 1 \\
\hline
\end{tabular}

Abbreviations: FDA, U.S. Food and Drug Administration; HIT-ME, health IT medication error; IV, intravenous.

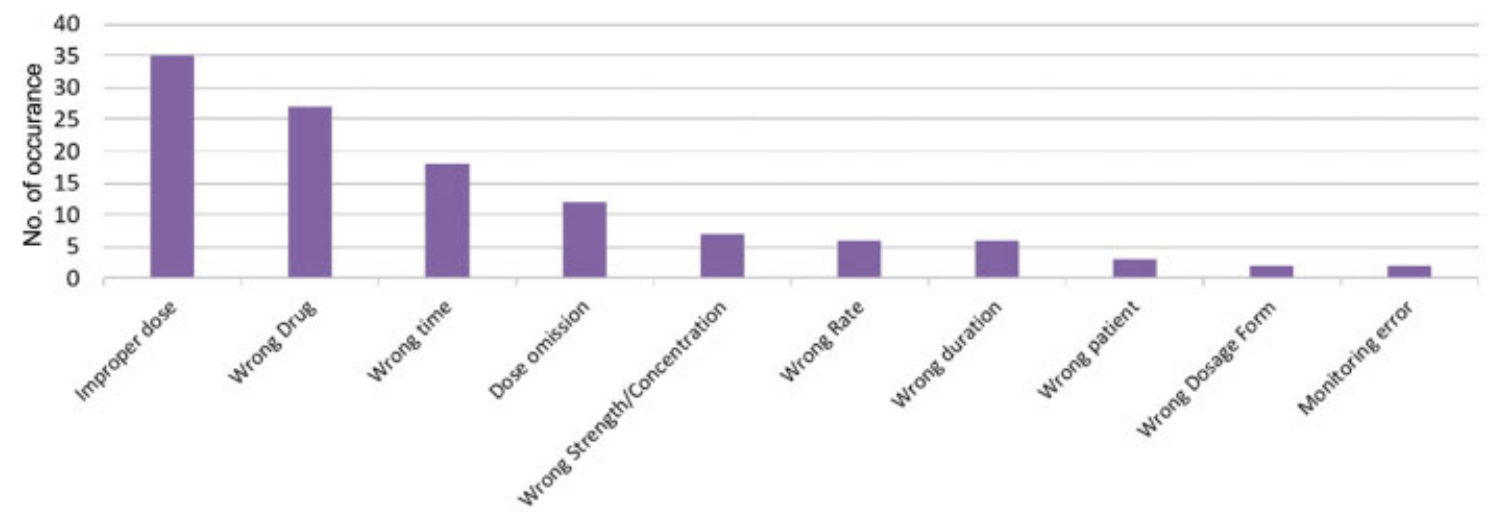

NCCMERP Error Types

Fig. 3 Distribution of involved error types. NCC MERP, National Coordinating Council for Medication Error Reporting and Prevention.

factors lead to receiving wrong information, missing information, receiving partial information, and delayed information, with the probabilities of $0.51,0.32,0.13$, and 0.04 , respectively. Receiving wrong information and missing information tend to cause the commission errors in decisionmaking with the probabilities of 0.62 and 0.64 , respectively.
Twenty-five percent of the wrong information events were detected before affecting patient outcomes. Making a wrong decision was more likely to cause wrong care process (probability: 0.89) and finally cause patient harm (probability: 0.94); patient harm could only be caused by altered care process, including a wrong care process and a delayed care 


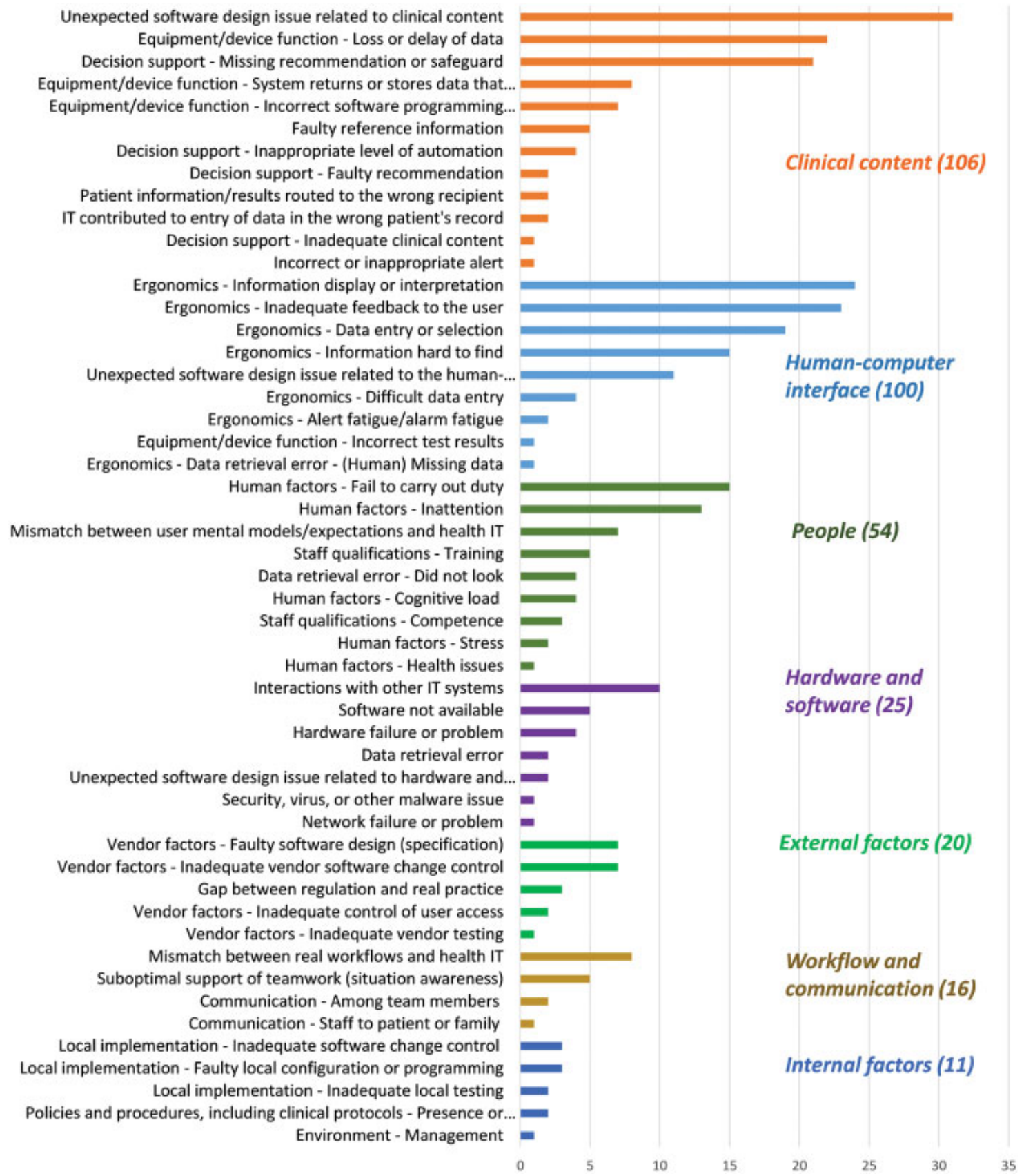

Fig. 4 Distribution of health information technology related contributing factors in 152 health IT medication error reports. The contributing factors are organized using Sittig and Singh's sociotechnical model. The number in each dimension indicates the count of matches of related contributing factors within the corresponding dimension.

process. A delayed decision led to delayed care process (probability: 1.0). Most cases in altered care processes ended with patient harm (probability: 0.94 and 0.9 ).

\section{Discussion and Future Work}

\section{The MAUDE Report Quality}

\section{A Rich Diversity of Health IT Related Reports in the MAUDE Database}

In this study, the identified 152 HIT-ME reports cover all event types in the patient safety event classification defined by the AHRQ Common Formats. The reports also cover 76.92\% medication error types in the NCC MERP taxonomy, $64.93 \%$ health IT related contributing factors in the composite classification, seven out of eight dimensions of the sociotechnical model, and 23 types of medical devices in the FDA product classification. The sources of reports include manufacturers, physicians, pharmacists, nurses, and patients. The aforementioned figures indicate a rich diversity of the MAUDE database and show that the MAUDE database is a resource for the nature and safety implications of health IT problems. ${ }^{21}$ The proportion of health IT related reports is expected to grow according to the observed increment of 


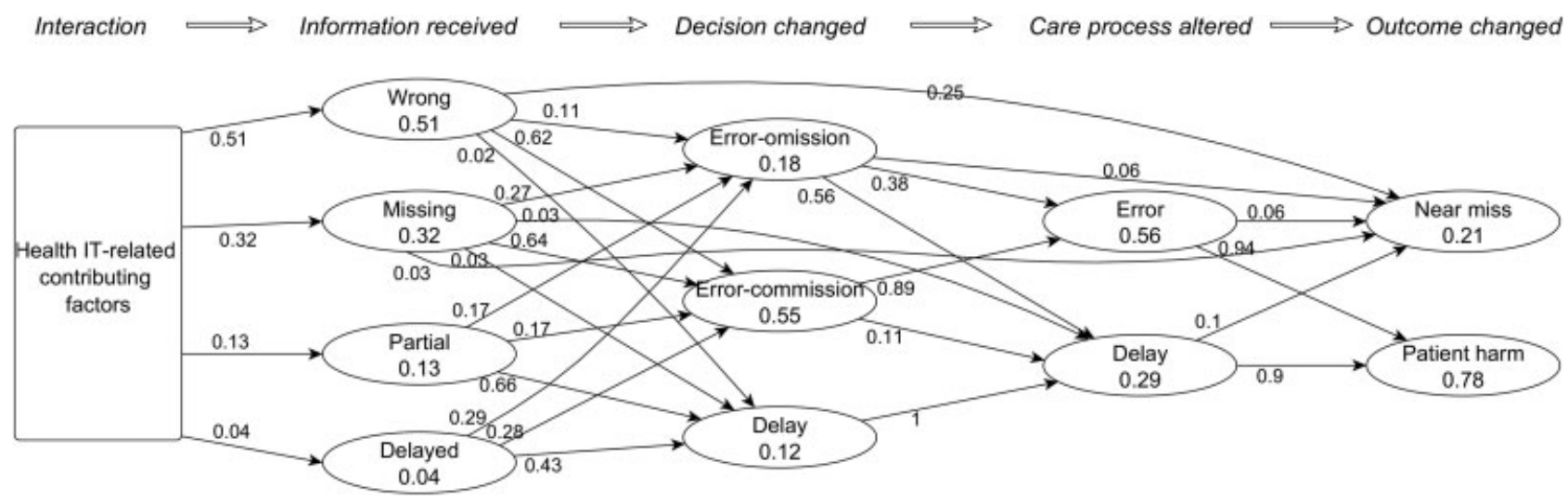

Fig. 5 The effects of health IT on medication safety from the viewpoint of information value chain. The transition probability at each effect transition line (directed line) denotes possibility of the transition from one effect to the other. IT, information technology.

incoming reports and the pervasive application of new health IT devices.

\section{Coded Categories of Medical Devices}

The FDA assigns a generic category of device by using the product codes. The type of medical device involved in the identified HIT-ME reports (-Table 2) exhibits that most devices are related to information processing. Thus, the product codes provide another opportunity to retrieve data effectively. Specialists from different medical areas could retrieve reports according to their interests by selecting targeted product codes. Some medical devices represented by product codes are occasionally associated with HIT-ME reports, meaning that sporadic events spread in a wide range could be an indicator for patient safety experts to explore.

\section{Demand for Quality Assessment}

The length of HIT-ME reports reflects a considerable amount of narratives in the identified reports. Based on our review, the narratives would be the most informative part in investigating the root cause and the effect. A typical narrative first describes the phenomenon, then the causal procedure, and then the underlying cause if applicable. In some cases, the narrative also provides information about what responses have been received from the vendor if it is a manufacturer report. However, the report qualities vary, which may bring a bias when reporters describe the events. Some reports merely describe the occurrence of the event without any information about the cause or the causal relationship. Therefore, a systematic assessment method is in need for identification of high-quality report and exploration of learning values of the historical reports.

\section{More Views Imply a Comprehensive Insight}

We investigated health IT induced safety reports related to medication errors through two views: (1) a composite classification of contributing factors guided by Sittig and Singh's sociotechnical model and (2) Coiera's information value chain. These two conceptual frameworks provide an opportunity to understand contexts and impacts of health IT induced medication safety reports, that is, from causes to outcomes. The sociotechnical model focuses on causal factors of event occurrence, whereas the information value chain focuses on the health care process and patient outcomes that might be affected by the errors or unsafe conditions due to the use of health IT products.

\section{Implication of the Results through the Sociotechnical Model}

To obtain the view of HIT-ME reports through the sociotechnical model, we plotted the distribution of health IT related contributing factors identified in the reports. The results indicate that (1) a variety of contributing factors (associated with seven out of eight dimensions) exhibit in the HIT-ME reports retrieved from the MAUDE database, (2) contributing factors are mostly associated with the clinical content followed by human-computer interface and people, and (3) organizational and system-related contributing factors are uncommonly reflected in the reports ( - Fig. 4).

The contributing factors associated with the technical dimensions, such as clinical content and human-computer interface, more frequently appeared in the HIT-ME reports. In addition, considerable human factors and organizational factors have also been manifested, such as communication, teamwork, and supervision support. Organizational and system-related factors may play underlying roles in causing a medication error. Additional tools such as the Hospital Survey on Patient Safety Culture ${ }^{33}$ would be helpful in identifying defects in a health care setting.

\section{Implication of the Results through the Information Value Chain}

Based on the reports interpreted by the information value chain, we obtained an overview of the effects of health IT related contributing factors on medication safety events (-Fig. 5). The presented view provides a way of showing how likely certain effect may be brought on one step and how it affects the other adjacent steps. For instance, receiving wrong information causes making a wrong decision with a probability of 0.62 , and the wrong information may lead to a wrong (probability: 0.38 ) or delayed (probability: 0.56 ) care process, with a high chance of resulting in patient harm 
(probability: 0.94). The information value chain may serve as a tool that can describe the effect of health IT induced medication errors.

\section{Implication of the Two Conceptual Frameworks}

The two views may serve as a profile of health IT induced medication safety events. The combined view based on the two conceptual frameworks may enhance the understanding of health IT induced problems and identification of the most significant risks to patient safety. The application of the two conceptual frameworks to the MAUDE database may be a general approach to analyze any event reports in the context of health IT. A full view would also have implication on developing a knowledge base supported by case-based reasoning. For example, when an e-MAR designer needs to know whether a problem relating to the selection of the drop-down menu of their product has happened before and what the consequences are, ${ }^{34}$ the knowledge base could facilitate a faceted search for this demand based on the contributing factors, effects on health care delivery, and patient outcomes. Besides, the application of the two conceptual frameworks may suggest a formalized reporting template for health IT induced medication errors. Reporting an event following the causal relationship, from contributing factors to effects, may enhance the completeness, quality, and utility of such reports.

\section{Limitations}

The sampled HIT-ME reports may not perfectly reflect a full picture of health IT induced medication safety events. A larger sample size is expected in future studies when exploring the periodically updated FDA MAUDE database. The causal relationship among contributing factors was not yet taken into account, which can be a direction for further investigations for a better understanding of how an event could happen under multiple contributing factors.

\section{Conclusion}

In this study, we investigated the publicly accessible reports on health IT induced medication safety events through two views, that is, Sittig and Singh's sociotechnical model and Coiera's information value chain. The two frameworks provide an opportunity to understand the comprehensive context of safety events and the impact of health IT induced errors on medication safety. The sociotechnical model helps identify the aspects causing a medication safety event. The information value chain helps uncover the effect of the health IT induced medication errors on health care process and patient outcomes. The findings suggest that the two views may serve as a new descriptive and analytical way in understanding health IT induced medication safety events.

\section{Clinical Relevance Statement}

The results of the views of the two conceptual frameworks may become a new descriptive and analytical way of understanding health IT induced medication safety events. The results help health care professionals better understand the context of the encountered medication safety events. The views presented under the two frameworks would help safety experts build up a knowledge base that can support case-based reasoning.

\section{Multiple Choice Questions}

1. What can be the causes of a medication error?
a. Only software issues.
b. Only mistakes by practitioners.
c. Only failure of institutional management.
d. All of the above.

Correct Answer: The correct answer is option d.

2. Health IT solutions can cause medication errors by means of ___?

a. Inconvenient human-computer interaction.

b. Information issues (e.g., wrong, missing, and delayed).

c. Affecting decision-making.

d. All of the above.

Correct Answer: The correct answer is option d.

Protection of Human and Animal Subjects

No human/animal subjects were involved in the project.

Funding

This project is supported by the Agency for Healthcare Research and Quality (grant number R01HS022895) and the UTHealth Innovation for Cancer Prevention Research Training Program Postdoctoral Fellowship (Cancer Prevention and Research Institute of Texas; grant \#RP160015). The content is solely the responsibility of the authors and does not necessarily represent the official views of the Agency for Healthcare Research and Quality.

Conflict of Interest

None declared.

\section{References}

1 Medication without Harm-Global Patient Safety Challenge on Medication Safety. Geneva: World Health Organization; 2017

2 Medicine Io. Preventing Medication Errors. Washington, DC: The National Academies Press; 2007

3 Sittig DF, Singh H. Defining health information technology-related errors: new developments since to err is human. Arch Intern Med 2011;171(14):1281-1284

4 Bates DW, Gawande AA. Improving safety with information technology. N Engl J Med 2003;348(25):2526-2534

5 Radley DC, Wasserman MR, Olsho LEW, Shoemaker SJ, Spranca MD, Bradshaw B. Reduction in medication errors in hospitals due to adoption of computerized provider order entry systems. J Am Med Inform Assoc 2013;20(03):470-476

6 Poon EG, Keohane CA, Yoon CS, et al. Effect of bar-code technology on the safety of medication administration. N Engl J Med 2010; 362(18):1698-1707

7 Khammarnia M, Kassani A, Eslahi M. The efficacy of patients' wristband bar-code on prevention of medical errors: A metaanalysis study. Appl Clin Inform 2015;6(04):716-727 
8 Rothschild JM, Lee TH, Bae T, Bates DW. Clinician use of a palmtop drug reference guide. J Am Med Inform Assoc 2002;9(03): 223-229

9 Ateya MB, Aiyagari R, Moran C, Singer K. Insulin bolus calculator in a pediatric hospital. Safety and user perceptions. Appl Clin Inform 2017;8(02):529-540

10 Koppel R, Metlay JP, Cohen A, et al. Role of computerized physician order entry systems in facilitating medication errors. JAMA 2005; 293(10):1197-1203

11 Castro GM, Buczkowski L, Hafner JM. the contribution of sociotechnical factors to health information technology-related sentinel events. Jt Comm J Qual Patient Saf 2016;42(02):70-76

12 Adler-Milstein J. Falling between the Cracks in the Software. Rockville, MD: AHRQ PSNet; 2016. Available at: https://psnet. ahrq.gov/webmm/case/382/falling-between-the-cracks-in-thesoftware. Accessed January 15, 2018

13 Adams KT, Howe JL, Fong A, et al. An analysis of patient safety incident reports associated with electronic health record interoperability. Appl Clin Inform 2017;8(02):593-602

14 Abramson E, Kaushal R. Situational (Un) Awareness. Rockville, MD: AHRQ PSNet; 2011. Available at: https://psnet.ahrq.gov/webmm/ case/249/situational-unawareness. Accessed January 22, 2018

15 Amato MG, Schiff GD. Slow Down: Right Drug, Wrong Formulation. Rockville, MD: AHRQ PSNet; 2018. Available at: https://psnet.ahrq. gov/webmm/case/432/slow-down-right-drug-wrong-formulation. Accessed February 08, 2018

16 Ashton EW. E-Prescribing: E for Error? Rockville, MD: AHRQ PSNet; 2012. Available at: https://psnet.ahrq.gov/webmm/case/ 260/e-prescribing-e-for-error. Accessed February 05, 2018

17 Wong A, Wright A, Seger DL, Amato MG, Fiskio JM, Bates D. Comparison of overridden medication-related clinical decision support in the intensive care unit between a commercial system and a legacy system. Appl Clin Inform 2017;8(03):866-879

18 Rehr CA, Wong A, Seger DL, Bates DW. Determining inappropriate medication alerts from "inaccurate warning" overrides in the intensive care unit. Appl Clin Inform 2018;9(02):268-274

19 Gong Y, Kang H, Wu X, Hua L. Enhancing patient safety event reporting: a systematic review of system design features. Appl Clin Inform 2017;8(03):893-909

20 Magrabi F, Ong M-S, Runciman W, Coiera E. An analysis of computer-related patient safety incidents to inform the development of a classification. J Am Med Inform Assoc 2010;17(06): 663-670
21 Magrabi F, Ong M-S, Runciman W, Coiera E. Using FDA reports to inform a classification for health information technology safety problems. J Am Med Inform Assoc 2012;19(01):45-53

22 Cheung K-C, van der Veen W, Bouvy ML, Wensing M, van den Bemt PMLA, de Smet PAGM. Classification of medication incidents associated with information technology. J Am Med Inform Assoc 2014;21(e1):e63-e70

23 Medicine Io. Health IT and Patient Safety: Building Safer Systems for Better Care. Washington, DC: The National Academies Press; 2012

24 Sittig DF, Singh $\mathrm{H}$. A new sociotechnical model for studying health information technology in complex adaptive healthcare systems. Qual Saf Health Care 2010;19(Suppl 3):i68-i74

25 Meeks DW, Smith MW, Taylor L, Sittig DF, Scott JM, Singh H. An analysis of electronic health record-related patient safety concerns. J Am Med Inform Assoc 2014;21(06):1053-1059

26 Coiera E. A new informatics geography. Yearb Med Inform 2016; (01):251-255

27 Kim MO, Coiera E, Magrabi F. Problems with health information technology and their effects on care delivery and patient outcomes: a systematic review. J Am Med Inform Assoc 2017;24(02): 246-250

28 Ash JS, Berg M, Coiera E. Some unintended consequences of information technology in health care: the nature of patient care information system-related errors. J Am Med Inform Assoc 2004;11(02):104-112

29 Kang H, Wang J, Yao B, Zhou S, Gong Y. Toward safer health care: a review strategy of FDA medical device adverse event database to identify and categorize health information technology related events. JAMIA Open 2018. Doi: 10.1093/jamiaopen/ooy042

30 Coiera E, Westbrook J, Wyatt J. The safety and quality of decision support systems. Yearb Med Inform 2006;1:20-25

31 Kobus DA, Amundson D, Moses JD, Rascona D, Gubler KD. A computerized medical incident reporting system for errors in the intensive care unit: initial evaluation of interrater agreement. Mil Med 2001;166(04):350-353

32 Fernald DH, Pace WD, Harris DM, West DR, Main DS, Westfall JM. Event reporting to a primary care patient safety reporting system: a report from the ASIPS collaborative. Ann Fam Med 2004;2(04): 327-332

33 Kohn LT, Corrigan JM, Donaldson MS. To Err Is Human: Building a Safer Health System. Washington, DC: U.S. Institute of Medicine; 1999

34 Denecke K. Automatic analysis of critical incident reports: requirements and use cases. Stud Health Technol Inform 2016;223:85-92 